\title{
La tortilla tradicional: un contexto de significación para la matemática de la variación
}

\author{
The traditional tortilla: a significance context for the mathematics of variation
}

\author{
Fredy de la Cruz Urbina \\ Gabriela Buendía Ábalos
}

\begin{abstract}
RESUMEN
Abstract

Las actividades comunitarias resultan relevantes en el marco de los subsistemas educativos como el Telebachillerato chiapaneco. En este escrito se pretende considerar ese saber cultural y reconocer su papel epistemológico para proporcionar elementos para el diseño de actividades para el aula. Como resultado, la elaboración de la tortilla se muestra como un contexto de significación para la matemática del cambio y la variación. Esto precisó articular los elementos epistemológicos del cambio y la variación, sustentarlos a través del desarrollo de prácticas variacionales y favorecer el reconocimiento de un sistema de referencia variacional. La articulación de estos elementos con la actividad comunitaria se evidencia como un contexto de significación para la intervención didáctica en el aula de matemáticas.

Palabras clave: actividades comunitarias, bachillerato, prácticas variacionales.

The community activities are relevant in the framework of the educational subsystems such as the Telebachillerato from Chiapas. In this paper, the aim is to consider this cultural knowledge and to recognize its epistemological role to give elements in the design of some classroom activities. As a result, the making of the tortilla is shown as a context of significance for the mathematics of change and variation. This required articulating the epistemological elements of change and variation, supporting them through the development of variational practices and favoring the recognition of a variational reference system. The articulation of these elements with community activity is evidenced as a context of significance for the didactic intervention in the mathematics classroom.

Keywords: community activities, high school, variational practices.
\end{abstract}




\section{INTRODUCCIÓN}

La experiencia docente en el subsistema de Telebachillerato en Chiapas en varias comunidades y regiones del estado ha permitido conocer que los jóvenes estudiantes realizan actividades diversas de tipo social, cultural, productivo, ritual y recreativo que, por lo general, aprenden por experiencia y autoridad paterna-materna en el seno familiar y comunitario.

De acuerdo con Martinez (2015), Rendón y Ballesteros (2003) y Díaz (2001), estas actividades manifiestan la comunalidad y se caracterizan por los siguientes rasgos: respeto a la tierra como madre y territorio, el trabajo colectivo, el consenso en asamblea, el servicio gratuito, los ritos y ceremonias. Cada actividad tiene como fin social el goce o disfrute para el bien común o el buen vivir. A través de la práctica y la constancia de su participación en dichas actividades, los alumnos desarrollan conocimientos y habilidades para ejecutarlas de manera eficiente. Por consiguiente, el alumnado posee ese conocimiento que es usado en diversas situaciones de su cotidiano. Denominaremos actividades comunales o comunitarias a las actividades que se distinguen por estos elementos.

Este escrito parte de considerar el aporte de estas actividades comunitarias hacia la matemática escolar en el Telebachillerato chiapaneco. En particular, se propone trabajar con la actividad comunitaria de elaboración de la tortilla hecha a mano o tradicional. El objetivo es presentarla como una base para generar un conocimiento matemático socioculturalmente significativo; esto será un contexto de significación, en particular, para la matemática del cambio y la variación.

\section{Actividades comunales y la matemática escolar}

Reconocemos que la escuela es normada por un discurso matemático escolar que, entre otras cosas, especifica los contenidos matemáticos y la manera como deben desarrollarse en el aula (Reyes-Gasperini, 2016). Por otro lado, existe un alumnado que posee

Fredy de la Cruz Urbina. Profesor de Telebachillerato en el estado de Chiapas, México. Actualmente es estudiante de doctorado en Matemática Educativa en el Centro de Investigación de Ciencia Aplicada y Tecnología Avanzada del IPN, México. Tema de investigación: "Un estudio socioepistemológico en el Telebachillerato chiapaneco, hacia un discurso matemático escolar inclusivo”. Es miembro del Grupo de Difusión de la Investigación en Matemática Educativa (DIME). Correo electrónico: frecu@, hotmail.com. ID: https://orcid.org/0000-0001-8461-0970.

Gabriela Buendía Ábalos. Red de Centros de Investigación en Matemática Educativa, México. Es docente de matemáticas de nivel medio superior a superior. Actualmente su campo de desarrollo es en la profesionalización docente en el área de Matemáticas, siendo asesora de varios programas institucionales: CGFIE-IPN, PIDPDM-CINVESTAV, especialidad en Didáctica de las Matemáticas-UNACH, entre otros. Es miembro del Comité Latinoamericano de Matemática Educativa (CLAME) y de la Red de Centros de Investigación en Matemática Educativa de México (Cimates). Es editora de la revista Investigación e Innovación en Matemática Educativa de la Red CimATEs. Correo electrónico: buendiag@hotmail.com. ID: https:/ /orcid.org/ 0000-0002-9456-4469. 
habilidades y conocimientos que se manifiestan en las actividades sociales, culturales, productivas y recreativas que lleva a cabo en su territorio socionatural comunitario (Sartorello y Bertely, 2019). Ahora bien, en ocasiones la escuela suele no considerar ciertos factores socioculturales, específicamente nos referimos a las actividades comunitarias que asumimos como posible fuente de significación del conocimiento, pues en ellas los alumnos tienen la oportunidad de aprender, comprobar, compartir y reconstruir su conocimiento en las diferentes actividades que realizan cotidiana o temporalmente en espacios vivos y reales (Bertely, 2019; Sartorello y Bertely, 2019).

Como una alternativa, este trabajo propone generar un diálogo entre la escuela y la comunidad, esto es, traer al aula de matemáticas elementos presentes en las actividades comunitarias para que sean base de significación del conocimiento matemático. Este diálogo permitirá que los estudiantes tengan la oportunidad de construir y dar significado a la luz de sus saberes al conocimiento matemático del cambio y la variación. Esto se considera factible a través de la noción de uso del conocimiento que plantea el enfoque socioepistemológico. De acuerdo con Cantoral (2013a), el uso refiere a la funcionalidad del conocimiento en situaciones específicas y está ligado al usuario y al contexto. Precisamente en la actividad comunitaria elaboración de la tortilla se visualizan usos del conocimiento matemático relativos al cambio y la variación que pueden dotar de significados a la matemática escolar. Ese es pues el objetivo de este escrito: dar evidencia de cómo una actividad comunitaria puede ser fuente de significación para la matemática escolar.

\section{Actividad comunitaria: la elaboración de la tortilla}

En México, el maíz y las tortillas por su consumo generalizado representan un símbolo de identidad que atraviesa conceptos de clase, etnia y raza. Su relevancia es más evidente en las comunidades rurales donde la gente sigue cultivando el maíz y consumiendo tortillas a la usanza tradicional. En Chiapas diversos mitos indígenas denotan al maíz como un alimento sagrado (López, Martínez y Sovilla, 2015).

La tortilla hecha a mano puede caracterizarse a partir de los rasgos de la comunalidad que reportan Rendón y Ballesteros (2003) porque implica el trabajo comunal con la milpa; esto involucra una relación de dependencia con la madre tierra, la cosmovisión, el uso de tecnologías y la educación como algo necesario para perpetuar el conocimiento que posee dicha comunidad para un fin social. Desde esta perspectiva la preparación de alimentos se considera como un rasgo cultural tecnológico que sostiene la vida individual y familiar dentro de la comunidad.

De acuerdo con la división del trabajo como rasgo comunal, los jóvenes participan en las actividades comunitarias según la edad y sexo. La elaboración de la tortilla es parte de las tareas que realizan las mujeres en las comunidades rurales chiapanecas. Las jóvenes de Telebachillerato regularmente llevan a cabo esta actividad que han 
aprendido desde niñas. Muchas de ellas ayudan a hacer las tortillas o tortear antes de ir a la escuela. Por su parte los varones trabajan en la milpa para cosechar el maíz y como parte de sus tareas también consiguen la leña para cocinar los alimentos.

A partir de la observación personal y testimonios de quienes elaboran tortillas, para su elaboración se sigue el siguiente proceso: en primer lugar se prepara la masa; esta debe tener una consistencia plástica, para ello se agrega empíricamente la cantidad de agua necesaria hasta hacerla moldeable. Como siguiente paso, se elige una porción de masa con los dedos, se le da una forma ovalada y después apoyándose en una base firme y sobre una pieza de plástico o papel se palmea, esto es, se dan golpes con las manos para darle forma y tamaño a la masa. Para finalizar, se pone sobre el comal para su cocción. El producto obtenido es lo que se denomina "tortilla".

El comal debe precalentarse para la cocción de la tortilla; por lo general se usa un fogón o cocina rústica. Se emplea la leña o carbón para lograr que el comal se ponga muy caliente. Entonces, se pone la tortilla y se deja por un tiempo, para después voltearla; se espera otro tiempo y se vuelve a voltear. De estas acciones de "voltear" depende que se produzca "una buena tortilla". La acción de "voltear" provoca que se genere una membrana o panza en la tortilla y que esta se infle, indicando su cocción óptima. Esta es la principal característica de una "buena tortilla", además determina el momento en que esta debe retirarse del comal. El proceso de cocción se realiza en uno a dos minutos aproximadamente (ver figura 1).
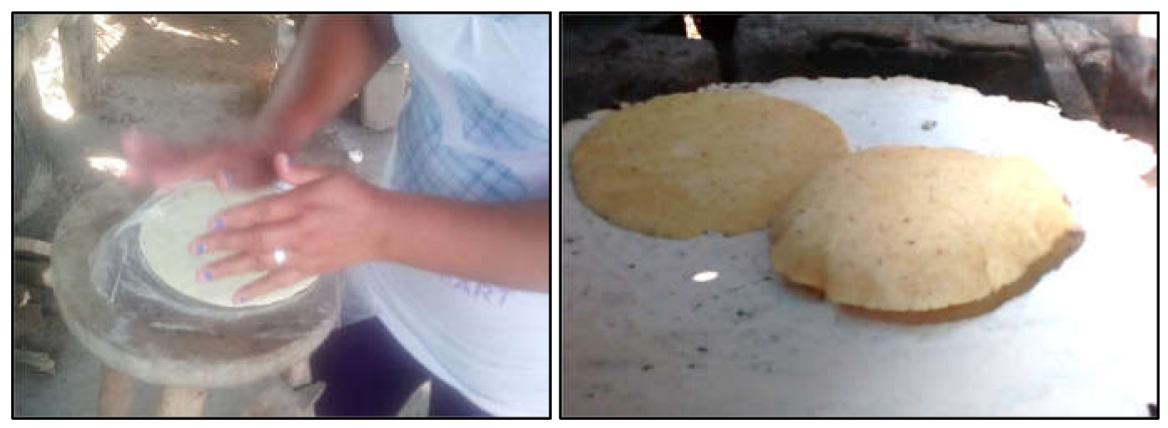

Figura 1. Elaboración y cocción de la tortilla.

Fuente: Elaboración propia.

El tiempo de cocción de la tortilla depende en gran medida de la temperatura del comal. Una ama de casa manifestó que depende "de cómo esté la lumbre o fuego; si el comal no está muy caliente la tortilla tarda más en cocerse y casi no sube [refiriéndose a la pancita] y si está muy caliente se quema, debe estar de buen tanto". Las personas que elaboran tortillas regulan el calor del comal, retirando o poniendo más leña al fogón.

En este proceso, la cocción de la tortilla es el escenario de interés para el estudio del cambio y la variación. ¿Cuándo se debe voltear la tortilla? La respuesta exige del 
reconocimiento del cambio de color y textura de la tortilla. La valoración de dicho cambio la realizan quienes han desarrollado conocimiento a través de la experiencia.

\section{Hacia la matemática escolar}

Nos proponemos considerar este saber popular para darle significado a la matemática escolar; en particular, el foco está en las acciones de voltear la tortilla que demandan la percepción y cuantificación empírica del cambio y, además, el análisis de dicho cambio a través de indicadores como el color, textura e inflado de la tortilla que suceden en este fenómeno endotérmico. Dicho fenómeno puede caracterizarse como una absorción de calor que produce una pérdida de humedad; en consecuencia, se genera la evaporación de agua provocando que la tortilla se infle por la liberación del hidrógeno y oxígeno (Valderrábano, 2005; Vera, 2007).

Identificamos que la medición empírica de la temperatura y el tiempo permiten realizar acciones y tomar decisiones durante el proceso de cocción para producir una buena tortilla. El cambio presente en este fenómeno endotérmico es valorado de manera cualitativa mediante dos técnicas.

\section{Técnica 1: visualizar el cambio de color.}

La persona observa que la tortilla cambia de color, lo cual se asocia con la humedad de la tortilla. Esta técnica se usa en el primer momento de voltear la tortilla, debe realizarse antes de que se seque por completo. Esto es, la persona observa la orilla de la tortilla antes de que comience a tomar un color café o más oscuro y el centro aún se vea húmedo o todavía presente el color natural (el color depende del tipo de maíz, por lo regular se usa más el maíz blanco y amarillo). Lo que sucede según Valderrábano (2005) es que, al añadir calor, el almidón que contiene la masa se gelatiniza por completo generando una membrana impermeable, esto impide que el vapor de agua se escape después de realizar el segundo momento de volteo.

\section{Técnica 2: sentir la textura.}

Esta técnica se utiliza en el segundo momento de volteo. La persona levanta un poco la tortilla y valora su textura, con ello comprueba que la tortilla ya esté cocida. En otras palabras, "siente que ya está seca", y esto permite realizar la segunda acción de voltearla. En este particular momento se observa que la valoración se hace en un área de la tortilla y con ello se infiere si ya está cocida por completo. Después de que se ha volteado, la tortilla permanece en el comal por un tiempo hasta que comienza a inflar la pancita. Cuando la persona observa esto, da ligeros golpecitos a la pancita con la yema de los dedos favoreciendo el desprendimiento en toda la tortilla, y la retira del comal. 
En síntesis, este escrito busca articular dichas técnicas visuales-táctiles y los conocimientos empíricos que viven en la elaboración de la tortilla. Concretamente, relacionar dicho fenómeno endotérmico con los conocimientos escolarizados de la matemática del cambio y la variación, para con ello favorecer su significación.

\section{Aspectos TEÓRICOS}

\section{Socioepistemología y los contextos de significación}

Cantoral, Montiel y Reyes-Gasperini (2015) mencionan que la socioepistemología, como marco teórico al seno de la matemática educativa, asume que el conocimiento matemático "tiene un origen y una función social asociados a un conjunto de actividades prácticas socialmente valoradas y normadas" (p. 12). Los autores comentan que este marco propone generar significaciones del conocimiento matemático a través de la problematización del saber situándolo en el entorno de la vida del estudiante; es decir, la cultura, los conocimientos y saberes, la historia y el presente del aprendiz se vuelven aspectos relevantes a considerar en los diseños didácticos. Por ello, el significado que las personas den al conocimiento matemático estará condicionado por las circunstancias socioculturales (Montiel y Buendía, 2012).

De acuerdo con Reyes-Gasperini (2016), la problematización del saber matemático con este enfoque se basa en el uso y las prácticas asociadas a los objetos matemáticos, los cuales constituyen la base epistemológica para su significación. Según la autora, esto plantea la necesidad de que profesores y alumnos experimenten un cambio de relación con el conocimiento matemático, esto es, la significación pasa de ser vista como atributo del objeto a otra derivada de su valor de uso. Así, los conceptos matemáticos toman otros matices según el marco de referencia donde se usan y de la relación de uso que se establezca entre el individuo y el conocimiento matemático (Cantoral, Moreno-Durazo y Caballero-Pérez, 2018).

Entonces, para la socioepistemología la significación está ligada al uso del conocimiento en una situación específica en la cual el contexto es fundamental. De acuerdo con Espinoza (2009), la significación es producto de la relación entre tres dimensiones: la situacional, la sociocultural y la racionalidad. Estas dimensiones conforman un contexto de significación. A partir del aporte de Espinoza (2009), se interpreta en este trabajo a la dimensión situacional como el conjunto de factores o circunstancias del espacio y tiempo en que se desarrolla la actividad. La sociocultural tiene que ver con factores culturales de la comunidad a la que pertenecen los actores. La racionalidad refiere a la funcionalidad del conocimiento matemático permeado por la dimensión situacional y sociocultural. En consecuencia, la naturaleza epistemológica del conocimiento queda ligada al contexto. Por ello, el contexto es fuente de significación del conocimiento matemático (Espinoza, 2014). 
Estas ideas se sostienen en tres principios socioepistemológicos, a saber: el principio de racionalidad contextualizada, el cual refiere que la relación entre el sujeto y el saber es una función del contexto; el principio del relativismo epistemológico sostiene que la validez del saber es subjetiva y relativa al individuo, del grupo cultural y al uso del conocimiento en un contexto específico, es decir, los resultados son validados con base en la coherencia de las argumentaciones contextuales realizadas, y el principio de resignificación progresiva alude a que la significación deriva de la acción del sujeto sobre el objeto y depende del escenario contextual donde se produce la acción (Cantoral, 2013a; Reyes-Gasperini, 2016).

Ball (2017) también reconoce que los diferentes ámbitos sociopolíticos, históricos, económicos, culturales, comunitarios y familiares influyen en la enseñanza de las matemáticas. Por su parte, Ingram (2018) argumenta que las acciones e interacciones del sujeto dependen de la comprensión que tenga del contexto donde actúa. Esto denota la relevancia que el contexto tiene para el aula de matemáticas.

Por otra parte, Tuyub y Buendía (2017) evidencian cómo una comunidad de ingenieros significa o encuentran sentido a lo que hacen en contextos socioculturales de significación que se caracterizan por tareas específicas en las que se manifiestan usos del conocimiento matemático, es decir, el conocimiento se utiliza con cierto significado. Para Reyes-Gasperini (2016) un contexto de significancia es aquel donde se favorece la significación del conocimiento mediante el uso y las prácticas asociadas a los objetos matemáticos.

En resumen, un contexto de significación será entonces un ámbito específico de la actividad humana que dota de sentido al conocimiento matemático a partir de su uso y funcionalidad en una situación específica, considerando las prácticas particulares en las que el ser humano, en tanto ente social, se involucra de manera intencional al hacer matemáticas. Se conforma por la dimensión situacional y sociocultural en donde vive o se pone en uso cierta racionalidad que puede ser popular, técnica o culta. Por tanto, se percibe como un referente para el aula de matemáticas.

\section{La matemática del cambio y la variación}

La matemática del cambio y la variación está presente en una amplia diversidad de procesos cuya pretensión es predecir el comportamiento futuro de un sistema (Cantoral, 2019, 2001). Tiene como intención comprender o interpretar una determinada situación de manera cuantitativa (Thompson, Carlson, Byerley y Hatfield, 2014). A partir del trabajo de Thompson y Carlson (2017), caracterizamos una situación variacional como aquella compuesta por magnitudes y relaciones entre magnitudes cuyos valores varían. Una magnitud es un atributo medible de la situación. 
Específicamente, el cambio tiene que ver con una modificación en el estado o condición que experimenta una magnitud en la situación, mientras que la variación implica el análisis de dicho cambio a través de la comparación, secuenciación, estimación y predicción; estas son consideradas prácticas variacionales (Cantoral, 2019; Caballero, 2018).

Cantoral (2001) comenta que la matemática del cambio y la variación tuvo como base contextos de significación ligados a fenómenos de la naturaleza en estrecha relación con los primeros elementos de la física clásica en el siglo XVII: el estudio del movimiento de los cuerpos en el espacio y la mecánica de medios continuos. El autor señala que fue a la luz de estos contextos que se "miraban a los fenómenos físicos desde la perspectiva de la matemática" (p. 77).

En el discurso escolar, el cambio y la variación requieren "la integración de distintos campos simbólicos, numéricos, algebraicos, analíticos, visuales, gráficos y geométricos, así como una adecuada comprensión de procesos matemáticos específicos, como: número, variable, constante, parámetro, función, límite, continuidad, derivada, integral, convergencia, representación e infinito" (Cantoral, 2013b, p. 45). Esto evidentemente muestra la importancia del cambio y la variación en los currículos escolares.

El objetivo de este trabajo busca generar significados a partir de la actividad comunitaria elaboración de la tortilla tradicional con estudiantes de Telebachillerato; la matemática del cambio y la variación es la que brinda herramientas para su análisis. En relación con este objetivo nos cuestionamos: ¿cómo las actividades comunitarias pueden contribuir en la construcción y significación de la matemática del cambio y la variación en el aula de matemáticas?

Al respecto, la teoría socioepistemológica considera que la predicción es la práctica social generadora del conocimiento referido a la variación y al cambio, lo cual es un fundamento epistemológico para el cálculo y el precálculo, ambos son ramas del conocimiento matemático referido al cambio y la variación que se empiezan a estudiar en el bachillerato. Cantoral (2019) señala que la necesidad de predecir es inherente al ser humano y exige de herramientas para su estudio, esto ante la incapacidad de adelantar el tiempo y con el objeto de conocer el estado ulterior de un sistema. Así, "la predicción consiste en la determinación de un estado desconocido de un fenómeno con base en el estudio sistemático y lógico del cambio y la variación que presentan las variables de estos fenómenos" (p. 111). Es entonces la práctica social la que funge de base y orientación en la explicación de los procesos de construcción social del conocimiento matemático (Cantoral, Reyes-Gasperini y Montiel, 2014). 


\section{La tortilla como un contexto de significación} para la matemática del cambio y la variación

En la socioepistemología, la incorporación de la dimensión social y cultural permite aceptar aspectos antes no considerados en el discurso escolar y que sirven de base para la significación del conocimiento (Cantoral, 2013a). En nuestro caso la elaboración de la tortilla se caracteriza como una situación variacional porque en ella intervienen variables que permiten comprenderla cuantitativamente, esto la hace factible para conformar un contexto de significación para la matemática del cambio y la variación. Asimismo, el fenómeno endotérmico que sucede en la cocción de la tortilla y los saberes propios que viven en ella hacen de esta actividad comunitaria un escenario que posibilita la transversalidad del conocimiento y la pluralidad epistemológica (Cordero, Gómez, Silva-Crocci y Soto, 2015).

En particular, la buena tortilla involucra un conocimiento funcional que quienes la elaboran han desarrollado a partir de las vivencias y experiencias con la actividad comunitaria. Respecto a cuándo se debe voltear la tortilla, puede entreverse de forma implícita la noción de predicción pues precisa de la valoración empírica del cambio y la cuantificación intuitiva del tiempo que se concreta en las dos acciones de volteo necesarias para producir la buena tortilla.

Así, el cambio y la variación presentes en el fenómeno endotérmico conjugado con la experiencia comunitaria de la actividad hacen factible que la elaboración de la buena tortilla pueda ser considerada un contexto de significación, objetivo que se pretende evidenciar a continuación. De ser así, este saber propio de la actividad comunitaria articulado con una epistemología de prácticas del cambio y la variación puede dotar de sentido a la matemática escolar.

\section{Aspectos metodológicos}

Asumimos entonces que la matemática del cambio y la variación es una construcción social y que se significa mediante el uso situado socioculturalmente. A fin de caracterizar a la elaboración de una buena tortilla como un contexto de significación, se recurrió al esquema metodológico propuesto por Montiel y Buendía (2012), que además de servir de ruta a la investigación provee una estructura hacia la intervención didáctica basada en la investigación. Específicamente, hemos considerado solo tres momentos o fases que nos marcan nuestra metodología: la problemática, epistemología de prácticas y la situación problema. Además, las acciones relacionantes: análisis socioepistemológico y desarrollo intencional de prácticas (figura 2). 


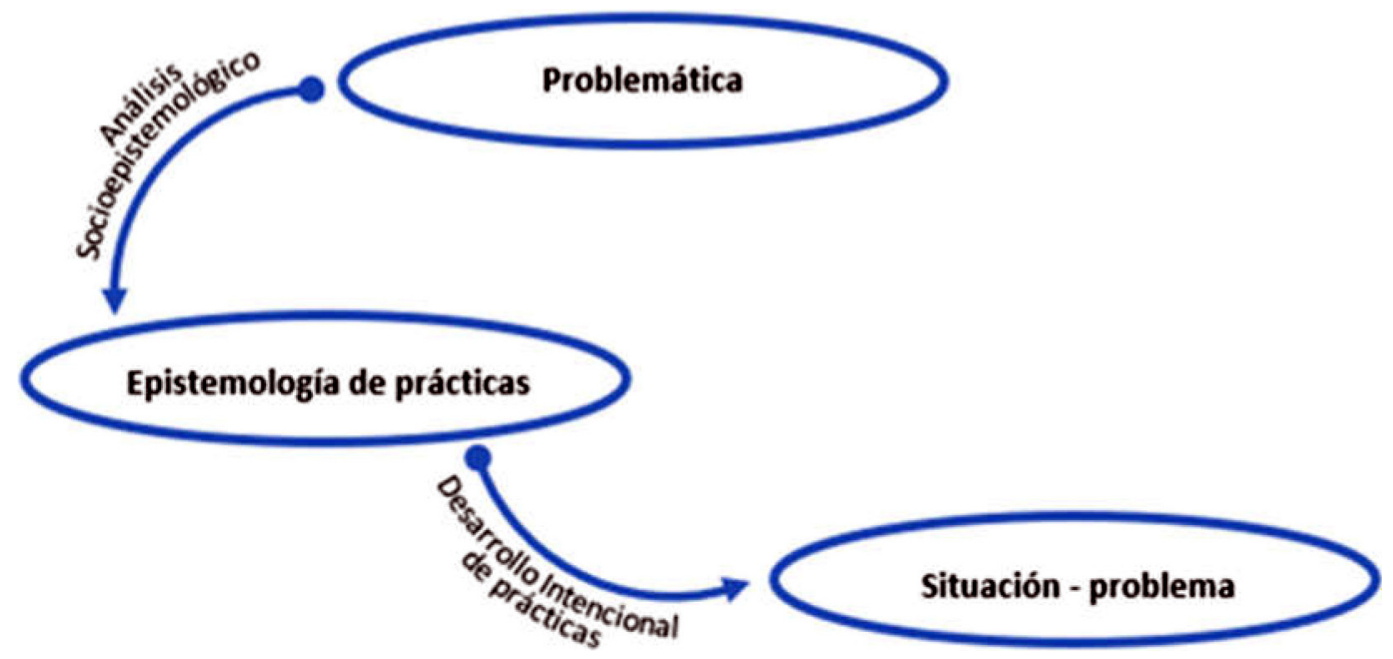

Figura 2. El esquema metodológico.

Fuente: Montiel y Buendía (2012).

La problemática que abordamos se resume en la pregunta “¿Cómo la elaboración de la tortilla puede conformar un contexto de significación para la matemática del cambio y la variación en el Telebachillerato chiapaneco? De acuerdo con las autoras, es necesario situar el saber a la vida de los estudiantes. Específicamente, el saber matemático se articula con aspectos sociales y culturales del alumnado.

Enseguida, de acuerdo con el esquema metodológico, es preciso hacer un análisis socioepistemológico. En nuestro caso se retoman los trabajos de Cantoral (2001, 2013a, 2019) y Caballero (2018), que han contribuido en la teorización del cambio y la variación desde la perspectiva de la construcción social del conocimiento. Con base en estos referentes se reconoce que la comparación, secuenciación, estimación y predicción, concebidas por los autores como prácticas variacionales, anteceden al conocimiento referido al cambio y la variación, por tanto, constituyen el sustento epistemológico para su significación.

El siguiente momento, epistemología de prácticas, pretende formular una explicación teórica de la problemática de estudio y esto servirá de base para la intervención didáctica. En este trabajo, dicha explicación conformará el contexto de significación que proponemos. Este constructo permite presentar a la actividad comunitaria elaboración de la tortilla como base de significación del conocimiento matemático. En ella convergen aspectos sociales y culturales en el fenómeno endotérmico, en el cual la matemática del cambio y la variación aporta herramientas para comprenderla de manera cuantitativa.

La acción relacionante de desarrollo intencional de prácticas (ver figura 2) consiste en favorecer las prácticas variacionales en la actividad comunitaria con el objetivo de 
propiciar la resignificación de la matemática del cambio y la variación. Es decir, los momentos anteriores permiten aterrizar en el diseño de la situación-problema. Una situación-problema es un conjunto de condiciones o instrucciones que permiten el desarrollo de acciones en el sistema didáctico (Montiel y Buendía, 2012).

Apoyados en este esquema metodológico, se presenta a continuación un contexto de significación y que además puede ser base para la intervención didáctica a través de una situación-problema. Es pues una herramienta para el estudio del cambio y la variación en situaciones variacionales que consta de tres momentos. En ella se ponen en juego las prácticas variacionales con la actividad comunitaria de "la buena tortilla".

El desarrollo intencional de dichas prácticas hacia el aula de matemáticas para conformar la situación-problema "la buena tortilla" permitirá hablar de los usos del conocimiento matemático y, en consecuencia, de su significación. Esta intencional se discute hacia el final del escrito, aunque el diseño final de alguna intervención didáctica (alguna situación de aprendizaje o situación-problema) no se presenta debido a que escapa del objetivo que se busca en este escrito: evidenciar una actividad comunal como fuente de significación para la matemática escolar.

\section{Conformando el contexto de significación}

Cantoral (2019) reporta que estudiar situaciones asociadas al cambio y la variación con fines predictivos precisa del establecimiento de una unidad de medida y un sistema de referencia variacional. Por su parte, Caballero (2018) revela que el sistema de referencia variacional se conforma por la relación que se establece entre la causalidad y la temporización. La primera está ligada a las preguntas ¿qué cambia? ¿respecto de qué cambia? y ¿por qué cambia de esa manera?, mientras que la temporización tiene que ver con analizar estados intermedios en la evolución de un fenómeno al responder a las preguntas ¿cuánto cambia y cómo cambia? Esto se relaciona con el reconocimiento de patrones y regularidad.

De acuerdo con González (2017) el reconocimiento de patrones alude a lo empírico y lo concreto de la situación, mientras que la regularidad corresponde a lo conceptual, a lo abstracto de la misma. El reconocimiento de patrones y regularidad es uno de los fines de las matemáticas y permite transitar a lo algebraico del fenómeno.

El estudio del cambio y la variación en situaciones variacionales requiere en primer lugar identificar las variables del fenómeno, entender cómo se cuantifican y se relacionan, requiere además conocer cómo cambian y se relacionan dichos cambios y sus variaciones (reconocer patrones y regularidad) y, más importante aún, comprender sus significados en la situación (Cantoral, Montiel y Reyes-Gasperini, 2015).

Con base en estos referentes, esta investigación configura tres momentos para el estudio del cambio y la variación en situaciones variacionales: 


\section{Momento I: Conocer el estado inicial del sistema}

Consiste en determinar aquellas magnitudes que describen con suficiente exactitud cierto estado del fenómeno. Esto requiere de un mecanismo de comparación y abstracción en el que es necesario establecer relaciones causales y una unidad de referencia para determinar cuáles son las variables significativas y cuáles pueden considerarse constantes. Responde a las preguntas ¿qué cambia? ¿respecto de qué cambia? y ¿por qué cambia de esa manera?

\section{Momento II: Reconocer patrones y regularidad}

Esto requiere de ciertas estrategias como establecer una unidad de medida, medir y experimentar. Enseguida, con los datos recopilados se produce una seriación de estados: construcción de tablas y gráficas. Después se analizan los datos y se reconocen patrones en la variación de los datos. Obedece a las preguntas ¿cuánto cambia? y ¿cómo cambia? Con ello se espera encontrar una medida del cambio y la manera como varía, por ejemplo: reconocer si es cada vez menor, es cada vez mayor, etc. Puede cuestionarse también si algo permanece constante o invariante.

\section{Momento III: Conformar un modelo predictivo}

A partir del análisis de las variaciones sucesivas de las variables obtenidas mediante la comparación y seriación, se establece un segundo mecanismo de abstracción que consiste en establecer los nexos funcionales entre variables y constantes. Responde a la pregunta ¿cuál es el modelo que organiza el comportamiento de los datos? Aquí juegan un papel fundamental la estimación y predicción como prácticas variacionales para hallar valores puntuales no contenidos en la tabla o gráfica (interpolación y extrapolación de datos) y, por último, encontrar el modelo predictivo que organiza la información.

En el caso de la buena tortilla la magnitud que permite la cuantificación del fenómeno endotérmico es la temperatura; esta experimenta cambios susceptibles para estudiar la variación por medio de la comparación, estimación, secuenciación y predicción, consideradas como prácticas variacionales (figura 3).

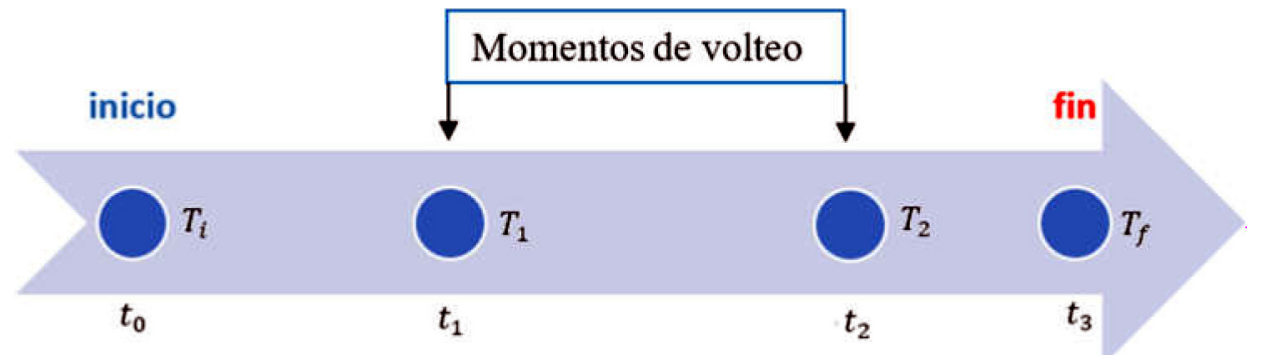

Figura 3. Cuantificación del fenómeno.

Fuente: Construcción personal. 
A priori, la temperatura inicial $T_{i}$ aumenta durante la cocción hasta $T_{1}$ en un tiempo $t_{1}$ cuando ocurre el primer momento de volteo. Posterior a ello la temperatura disminuye y después aumenta hasta $T_{2}$ en un tiempo $t_{2}$, cuando ocurre el segundo momento de volteo. Después de cierto tiempo $t_{3}$ la tor tilla comienza a "inflar" la pancita y es cuando se retira del comal con una temperatura $T_{f}$.

La significación del cambio y la variación en la buena tortilla se favorece con el estudio de la temperatura en esos estados específicos e intermedios a través de las prácticas variacionales en los diferentes momentos, por ejemplo:

- Comparar: se usa al analizar entre un estado y otro aquello que cambia y por qué cambia. Inicialmente esto se hace por medio de los sentidos: la percepción y reconocimiento del cambio de temperatura. En lo posterior, hace uso de técnicas como la diferenciación.

- Secuenciar o seriar: esto requiere recopilar datos de la variable mediante un proceso de medición y es útil para organizar y representar los datos en tablas y gráficas. Después, identificar por comparación cuánto cambia y cómo cambia la temperatura.

- Estimar: esta práctica se pone en uso al inferir valores no conocidos de la temperatura como producto de un análisis de los datos secuenciados en tablas o gráficas.

- Predecir: precisa de procesos de abstracción de la variable y sus relaciones para conformar un modelo que explique el comportamiento del fenómeno.

A partir de esta epistemología de prácticas se articula la actividad comunitaria hacia el aula de matemáticas y con ello se favorece una resignificación situada socioculturalmente de la matemática del cambio y la variación en el Telebachillerato chiapaneco.

Esta epistemología de prácticas permite considerar a la elaboración de la buena tortilla como un escenario de significación conformado por tres momentos; cada momento implica desarrollar ciertas tareas que se fundamentan en el reconocimiento de un sistema de referencia variacional como en el uso de prácticas variacionales. Además se consideran los conocimientos del alumnado y se promueve su participación en las distintas actividades. A continuación se describe cada uno de ellos.

Momento 1. Aquí se reflexiona sobre el proceso de cocción de la buena tortilla: qué cambia y qué produce el cambio. Este planteamiento procura identificar las magnitudes que están presentes en la situación y establecer relaciones concretas entre ellas y en lo que convenga evidenciarlo con la experiencia. Se promueve la reflexión a través de las preguntas ¿qué cambia durante la cocción de la tortilla?, ¿cómo se percibe dicho cambio?, ¿por qué cambia de esa manera? y ¿con respecto de qué cambia? Se espera que la discusión de estas preguntas permita reconocer a la temperatura y el tiempo como variables esenciales o significativas que permiten comprender el fenómeno endotérmico. 
Momento 2. Consiste en recopilar y analizar datos de la variable temperatura. Para este fin se considera el diseño de medios tecnológicos que faciliten la recolección de datos y que permitan la reflexión sobre las preguntas ¿cómo cambia y cuánto cambia la temperatura entre los momentos de volteo?, ¿cómo es la temperatura en el instante previo de voltear la tortilla $\left(T_{1}\right.$ y $\left.T_{2}\right)$ ? ¿qué ocurre después de voltear la tortilla?, ¿qué características presenta la gráfica al voltear la tortilla?, ¿qué características tiene la gráfica cuando la tortilla infla la pancita? Se espera que al analizar la secuencia de los datos se pueda argumentar cómo cambia y cuánto cambia la temperatura e identificar cuál es su comportamiento alrededor de la acción de voltear la tortilla.

Momento 3. Inicia con situaciones que requieren estimar la temperatura de la tortilla para valores no contenidos en la tabla. Esto precisa de analizar estados intermedios, es decir, temporizar. Se cuestiona: ¿qué pasa con $T$ de $t_{1}$ a $t_{2}$ y a $t_{3}$ ?, ¿cómo es la gráfica de una buena tortilla?, ¿todas las tortillas se cuecen de la misma manera?, ¿existe alguna relación entre las dos acciones de voltear la tortilla?, ¿cuál es la ecuación que describe la cocción de una buena tortilla? y ¿cuándo se debe voltear la tortilla? Esto pretende evidenciar la articulación de los argumentos construidos del cambio y la variación en la actividad comunitaria.

\section{Discusión}

\section{Una actividad comunitaria como contexto} de significación para la matemática escolar

A partir de la observación de la elaboración de la tortilla con amas de casa chiapanecas y la experimentación personal, se considera a la temperatura y al tiempo como variables que permiten cuantificar el fenómeno endotérmico y transitar de manera significativa de lo cotidiano a la matemática del cambio y la variación. Es decir, pasar de lo cualitativo a lo cuantitativo, y favorecer la matematización del fenómeno.

Dicho tránsito no se centra propiamente en objetos matemáticos sino en el desarrollo y reconocimiento intencional de prácticas en el contexto de significación, por ejemplo: medir el tiempo y la temperatura durante la cocción, secuenciar los datos recopilados y organizarlos en tablas y gráficas, comparar valores para cuantificar el cambio, comparar variables entre las acciones de volteo, estimar tiempos y temperaturas al momento de voltear la tortilla, predecir el comportamiento del fenómeno. Se busca relacionar lo cualitativo del fenómeno con lo cuantitativo poniendo en juego herramientas de la matemática del cambio y la variación que comprende el nivel de bachillerato, a saber: variable, constante, relación funcional, clasificación de funciones, dominio, formas de representación (numérica, gráfica, algebraica), plano cartesiano, pendiente, punto de inflexión, razón de cambio, variación, entre otras. La intención 
didáctica es favorecer un cambio de relación con el conocimiento matemático a partir de su uso, de donde deviene la significación (principio del relativismo epistemológico).

La significación en este contexto depende en gran medida de la experiencia del alumnado con la actividad comunitaria. Es decir, la cultura del alumnado es un aspecto relevante en el diseño (principio de racionalidad contextualizada). En la tabla 1 se presentan las acciones y prácticas que se ponen en juego en cada momento de la actividad y lo que se espera en cada una de ellas.

Para el estudio del cambio y la variación en la elaboración de la tortilla, primero es conveniente experimentar con la actividad comunitaria. Sigue un proceso de reflexión que consiste en reconocer el conjunto de magnitudes que intervienen en la situación y definir cuáles son las variables esenciales o significativas que permiten tener cierto control del fenómeno, es decir, pasar de lo cualitativo a lo cuantitativo (momento 1).

Tabla 1. Análisis de la propuesta.

\begin{tabular}{|c|c|c|}
\hline Momento & Acciones y prácticas en uso & Intencionalidad \\
\hline \multirow[t]{3}{*}{ Momento uno } & Experimentar & Reconocer técnicas y saberes locales \\
\hline & Comparar y seleccionar & Reconocer el cambio en el proceso de cocción \\
\hline & Analizar & Establecer las magnitudes y relaciones concretas del fenómeno \\
\hline \multirow[t]{3}{*}{ Momento dos } & Medir & Obtener datos de la cocción \\
\hline & Secuenciar y graficar & Representación del fenómeno \\
\hline & Comparar y estimar & Reconocer patrones de regularidad \\
\hline \multirow[t]{2}{*}{ Momento tres } & Comparar y estimar & Analizar estados intermedios (temporizar) \\
\hline & Estimar y predecir & Conformar un modelo predictivo \\
\hline
\end{tabular}

Fuente: Construcción personal.

Pasar de lo cualitativo a lo cuantitativo del fenómeno, no es trivial. Precisamente la matemática escolar propia del nivel bachillerato confluye como herramienta significativa para ello. $\mathrm{Al}$ respecto es necesario reflexionar en aquello que puede medirse o cuantificarse y cómo realizar el proceso de medición, así como la manera de organizar los datos. Requiere pensar en los recursos disponibles, sus alcances y limitaciones y en qué condiciones la medición tiene sentido. Esto implica asumir que ciertas magnitudes identificadas en el momento 1 serán consideradas constantes o cuasi-constantes, por ejemplo: la cantidad de masa en cada tortilla, la fuente de calor, etc.

El fenómeno de estudio que se ha caracterizado como endotérmico tiene aspectos cualitativos y cuantitativos. Los aspectos cualitativos que se consideran son los establecidos por la tradición y la experiencia. Lo cuantitativo se propicia con las herramientas teórico-metodológicas del cambio y la variación. Por la naturaleza del fenómeno endotérmico es factible considerar a la temperatura como una variable que permite argumentar sobre el fenómeno. 
El momento 3 consiste en realizar un análisis local y global del fenómeno por medio de la gráfica. Local es en el sentido de analizar el cambio entre estados, es decir, estimar el valor de la temperatura o bien el tiempo con ciertos valores puntuales conocidos. Pero también precisa de un análisis global que permita reconocer la manera como el cambio evoluciona, y a partir de estos elementos inferir el tipo de relación funcional que se presenta. Se espera que estas tareas sean base para transitar hacia un modelo algebraico de la situación.

En resumen, la elaboración de la tortilla hecha a mano resulta un contexto de significación para la matemática del cambio y la variación. Primero porque implica una situación variacional en donde está en juego un fin social: elaborar una buena tortilla; segundo, es una actividad propia de la comunidad y es parte del alumnado como un rasgo sociocultural de su contexto donde vive cierta racionalidad; tercero, permite poner en juego ciertas prácticas variacionales con la experiencia de la actividad comunitaria y, cuarto, la matemática del cambio y la variación propia del bachillerato aporta herramientas para su análisis.

\section{Intencionalidad de las prácticas: hacia una situación problema}

Un contexto de significación propuesto sobre una epistemología de prácticas y usos permite una intervención didáctica en la que el conocimiento matemático se resignifique a la luz de dichos elementos: es el conocimiento en uso. Así, una situaciónproblema se puede diseñar con base en los tres momentos anteriores y retomando las preguntas propuestas a manera de indicaciones por hacer.

Aunque la presentación de una situación-problema excede al objetivo planteado en este escrito, nos permitimos presentar un aporte concreto referido al momento 2 sobre la cuantificación de la temperatura. Para su cuantificación se confeccionó un dispositivo tecnológico integrado por una placa Arduino, un sensor infrarrojo de temperatura modelo MLX90614, cables jumper o Dupont, cable USB, computadora, controladores Arduino y software Excel (ver figura 4). Se optó por seguridad utilizar un sensor infrarrojo de temperatura y así evitar el menor contacto. El uso de Arduino y Excel se requiere para facilitar la visualización y manipulación de los datos, además de disponer de los datos en tiempo real y con cierta precisión durante todo el proceso. Estas son algunas razones del empleo de este dispositivo tecnológico, además representa un costo accesible.

Para realizar la medición (momento 2) es conveniente tener en cuenta ciertas consideraciones: la medición se realiza en el centro de la tortilla, se desprecian las interferencias que se producen al voltear la tortilla, se asume que la fuente de calor permanece constante y que la técnica o proceso para obtener una buena tortilla requiere dos acciones de voltear la tortilla. 


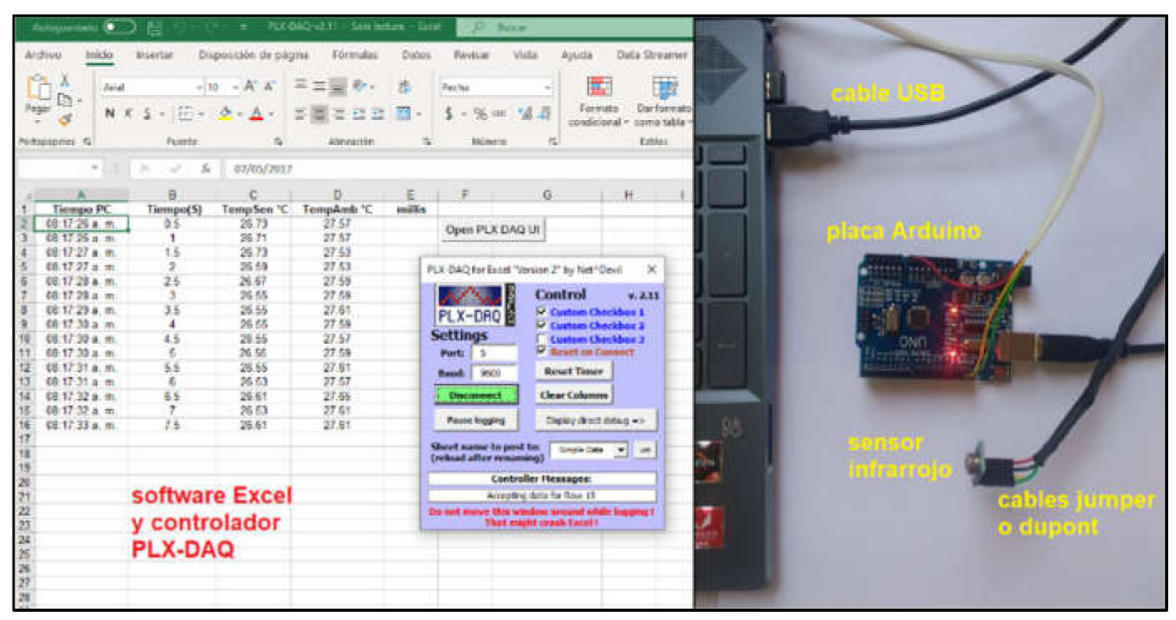

Figura 4. Dispositivo para la recolección de datos. Fuente: Elaboración propia.

En este proceso es importante que los alumnos se involucren en la medición de los datos, propongan estrategias de medición y realicen varias experimentaciones. Esto con el objetivo de reconocer patrones de regularidad, donde la actividad de medición tiene un rol importante en el momento 2.

\section{Comentarios Finales}

La elaboración de la tortilla que presentamos muestra la relevancia que tiene para la matemática escolar como fuente de significación del conocimiento. No obstante, no se trata solamente de realizar actividades comunitarias en la escuela pues su inclusión al aula de matemáticas requiere de una epistemología que permita su articulación y problematización con el conocimiento matemático; es decir, desarrollar un contexto de significación que provea elementos al estudiante para la construcción de significados. Decimos que la elaboración de la tortilla es un contexto de significación para el tratamiento de la matemática del cambio y la variación porque permite analizarlo, estudiarlo a través del fenómeno endotérmico que intencionalmente busca lograr una buena tortilla.

En concordancia con esto, la teoría socioepistemológica sostiene que el conocimiento matemático se resignifica a través de ciertas prácticas. En el caso de la matemática del cambio y la variación se considera que las prácticas de medir, comparar, secuenciar, estimar y predecir anteceden a este conocimiento. Con esta perspectiva la situación-problema, así como cualquier otra intervención didáctica con el mismo sustento epistemológico, no se centra propiamente en objetos matemáticos sino en el desarrollo intencional de prácticas. A la luz de este marco se comprendió una manera de estudiar y favorecer la matemática del cambio y la variación en y con la 
actividad comunitaria. Por ahora, el resultado es reconocer a la elaboración de la tortilla como un contexto de significación y haber reconocido la posibilidad de diseñar una situación-problema en ese marco. Esto hace que la intervención didáctica sea factible.

La elaboración de la tortilla como actividad comunitaria y contexto de significación para la matemática escolar puede ser una alternativa para desarrollar discursos inclusivos que favorezcan la construcción del conocimiento matemático desde su funcionalidad asociada a la vida cotidiana de los estudiantes. Por ello, consideramos que el contexto es fuente de significación del conocimiento matemático.

\section{REFERENCIAS}

Ball, D. L. (2017). Uncovering the special mathematical work of teaching. En G. Kaiser (ed.), Proceedings of the 13th International Congress on Mathematical Education, 13, 11-34. Gewerbestrasse, Switzerland: Springer Open. DOI: https://doi.org/10.1007/978-3-319-62597-3_2.

Bertely, M. (2019). Nuestro trabajo en las milpas educativas. Articulando e construindo saberes 4. DOI: https:// doi.org/10.5216/racs.v4.59219.

Caballero, M. A. (2018). Causalidad y temporización entre jóvenes de bachillerato. La construcción de la noción de variación y el desarrollo del pensamiento y lenguaje variacional [Tesis de doctorado no publicada]. Centro de Investigación y de Estudios Avanzados del IPN, México.

Cantoral, R. (2001). Matemática educativa. Un estudio de la formación social de la analiticidad. México: Grupo Editorial Iberoamérica.

Cantoral, R. (2013a). Teoría socioepistemológica de la matemática educativa. Estudios sobre construcción social del conocimiento. Barcelona: Gedisa.

Cantoral, R. (2013b). Desarrollo del pensamiento y lenguaje variacional. México: Subsecretaría de Educación Media Superior.

Cantoral, R. (2019). Caminos del saber. Pensamiento y lenguaje variacional. Barcelona: Gedisa.

Cantoral, R., Montiel, G., y Reyes-Gasperini, D. (2014). Hacia una educación que promueva el desarrollo del pensamiento matemático. Escri/viendo, Revista Pedagógica, 13(24), 19-28.

Cantoral, R., Montiel, G., y Reyes-Gasperini, D. (2015). El programa socioepistemológico de investigación en matemática educativa: el caso de Latinoamérica. Revista Latinoamericana de Investigación en Matemática Educativa, 18(1), 5-17.
Cantoral, R., Moreno-Durazo, A., y Caballero-Pérez, M. (2018). Socio-epistemological research on mathematical modelling: An empirical approach to teaching and learning. ZDM Mathematics Education, 50 (1), 77-89. DOI: https://doi.org/10.1007/s11858-0180922-8.

Cantoral, R., Reyes-Gasperini, D., y Montiel, G. (2014). Socioepistemología, matemáticas y realidad. Revista Latinoamericana de Etnomatemática, 7(3), 91-116.

Cordero, F., Gómez, K., Silva-Crocci, H., y Soto, D. (2015). El discurso matemático escolar: la adherencia, la exclusión y la opacidad. Barcelona: Gedisa.

Díaz, F. (2001, 12 mar.). Comunidad y comunalidad. La Jornada Semanal, 314. Recuperado de https://www. jornada.com.mx/2001/03/11/sem-cara.html.

López, J., Martínez, G., y Sovilla, B. (2015). Tortillas de maíz: simbolismo nacional e incremento de precios en un contexto de diferenciación social en el área urbana de San Cristóbal de las Casas, Chiapas. Espacio I + D Innovación más Desarrollo, 4 (8), 9-50. DOI: https://doi.org/10.31644/IMASD.8.2015.a01.

Espinoza, L. (2009). Una evolución de la analiticidad de las funciones en el siglo XIX. Un estudio socioepistemológico [Tesis de Maestría no publicada]. Cinvestav-IPN, México.

Espinoza, L. (2014). La desescolarización del saber: su construcción social desde el malabarismo y las artes circenses [Tesis doctoral no publicada]. Departamento de Matemática Educativa del Cinvestav-IPN, México.

González, A. (2017). Aspectos conceptuales y didácticos del pensamiento algebraico. Areté. Revista Digital del Doctorado en Educación de la Universidad Central de Venezuela, 3(5), 7-38. 
Ingram, J. (2018). Moving forward with ethnomethodological approaches to analyzing mathematics classroom interactions. ZDM Mathematics Education, 50(6), 1065-1075. DOI: https://doi.org/10.1007/ s11858-018-0951-3.

Martinez, J. (2015). Conocimiento y comunalidad. Bajo el Volcán, 15(23), 99-112. https://www.redalyc.org/ articulo.oa?id=286/28643473006.

Montiel, G., y Buendía, G. (2012). Un esquema metodológico para la investigación socioepistemológica: ejemplos e ilustraciones. En A. Rosas y A. Romo (eds.), Metodología en matemática educativa: visiones y reflexiones (pp. 61-88). México: Lectorum.

Rendón, J. J., y Ballesteros, M. (2003). La comunalidad: modo de vida en los pueblos indios. México: Dirección General de Culturas Populares e Indígenas.

Reyes-Gasperini, D. (2016). Empoderamiento docente y socioepistemología. Un estudio sobre la transformación educativa en matemáticas. Barcelona: Gedisa.

Sartorello, S., y Bertely, M. (2019). Milpas educativas para el buen vivir: nuestra cosecha. México: INIDE-IBERO y CIESAS.

Thompson, P. W., y Carlson, M. P. (2017). Variation, covariation, and functions: Foundational ways of thinking mathematically. En J. Cai (ed.), Compendium for research in mathematics education (pp. 421-456). Reston, VA: National Council of Teachers of Mathematics.

Thompson, P. W., Carlson, M. P., Byerley, C., y Hatfield, N. (2014). Schemes for thinking with magnitudes: A hypothesis about foundational reasoning abilities in algebra. En K. C. Moore, L. P. Steffe y L. L. Hatfield (eds.), Epistemic algebra students: Emerging models of students'algebraic knowing, WISDOMe Monographs (vol. 4, pp. 1-24). Laramie, WY: University of Wyoming. Recuperado de: http://bit.ly/1aNquwz.

Tuyub, I., y Buendía, G. (2017). Gráficas lineales: un proceso de significación a partir de su uso en ingeniería. IE Revista de Investigación Educativa de la REDIECH, $8(15), 11-28$.

Valderrábano, C. R. (2005). Propiedades físicas y químicas del grano, nixtamal, masa y tortillas elaboradas con maí germinado, y la evaluación sensorial de las tortillas [Tesis de Maestría no publicada]. Cinvestav, México.

Vera, M. I. (2007). Quimica general. Unidad VII: Termoquimica [Apuntes de clase]. Recuperado de: http://exa. unne.edu.ar/quimica/quimgeneral/temas_parciales/ Unidad_07_Termoqu_mica_2007.pdf.

Cómo citar este artículo:

De la Cruz Urbina, F., y Buendía Ábalos, G. (2021). La tortilla tradicional: un contexto de significación para la matemática de la variación. IE Revista de Investigación Educativa de la REDIECH, 12, e1098. doi: https://doi.org/10.33010/ ie_rie_rediech.v12i0.1098. 